\title{
Transpiração pelo método da sonda de dissipação térmica em floresta de transição Amazônica-Cerrado
}

\author{
Osvaldo B. Pinto Jr. ${ }^{1}$, George L. Vourlitis ${ }^{2}$, Luciana Sanches ${ }^{3}$, \\ Higo J. Dalmagro', Francisco de A. Lobo ${ }^{4} \&$ José de S. Nogueira ${ }^{1}$
}

\begin{abstract}
RESUMO
Com este trabalho objetivou-se analisar o comportamento do fluxo de seiva em espécies da floresta de transição Amazônia Cerrado e caracterizar a dependência do fluxo de seiva, em função do déficit de pressão de vapor da atmosfera (DPV). O fluxo de seiva foi medido utilizando-se sondas de dissipação térmica em 5 espécies diferentes. Os dados foram divididos em quatro estações. No período experimental as curvas de variação sazonal do fluxo de seiva evidenciam a ocorrência de picos no período úmido. Esta tendência sazonal do fluxo de seiva foi evidenciada pela relação entre valores diários de transpiração e do DPV. O valor limite do DPV nessas estações foi de 1 a 1,5 kPa. Com referência ao fluxo de seiva como representativo da taxa transpiratória das plantas, a redução do fluxo no período seco não confirma a hipótese da manutenção do processo de evapotranspiração. O fluxo de seiva nas espécies do estudo é influenciado mais pelas condições atmosféricas do que mesmo pela disponibilidade de água no solo, o que suscita um possível armazenamento da água no caule nos períodos mais secos quando a área foliar diminui mas a taxa transpiratória não é significativamente distinta daquela do período chuvoso.
\end{abstract}

Palavras-chave: fluxo de seiva, evapotranspiração, armazenamento de água

\section{Transpiration by the heat dissipation probe method in transition Amazon-Savannah forest}

\begin{abstract}
The objective of this study was to analyse the behavior of sap flow in a transition Amazon-Savannah forest species, to characterize the dependence of sap flow as a function of atmospheric vapor pressure deficit (VPD). The sap flow was measured using the technique proposed by Granier, using heat dissipation probes in five different species. The data were divided into four seasons. In the experimental period, the curves of seasonal variation of sap flow showed an occurrence of peaks in the wet season. This seasonal sap flow trend was evidenced by the relationship between daily values of transpiration and VPD. The threshold value of VPD at these stations ranged from 1 to $1.5 \mathrm{kPa}$. Considering sap flow as representative of plant transpiration, reduction of the flux in the dry season did not confirm the hypothesis of maintenance of evapotranspiration process. The sap flow in the studied species was influenced more by weather than soil water availability. This raises the possibility of water storage in the stem during drier periods, when leaf area decreases. However the transpiration rate is not significantly different from that of the rainy season.
\end{abstract}

Key words: sap flow, evapotranspiration, water storage

1 IF/UFMT, Av. Fernando Corrêa da Costa, 2367, Cidade Universitária, CEP 78060-900, Cuiabá, MT. Fone: (65) 3615-8735. E-mail: osvaldo.borges@gmail.com; higojdalmagro@gmail.com; nogueira@ufmt.br

${ }^{2}$ Department of Biological Sciences Hall 1- Room 320 California State University San Marcos, CA 92096, USA Phone: +55 760-750-4119. E-mail: georgev@csusm.edu

${ }^{3}$ Departamento de Engenharia Sanitária e Ambiental/UFMT. Fone: (65) 3615-8738. E-mail: Isanches@ufmt.br

${ }^{4}$ Departamento de Solos e Engenharia Rural/UFMT. Fone: (65) 3615-8682. E-mail: fdalobo@gmail.com 


\section{INTRODUÇÃO}

Nas duas últimas décadas algumas técnicas, conhecidas como "métodos térmicos", vêm sendo testadas em que, apesar da complexidade envolvida, vêm demonstrando bons resultados para estimativas da transpiração de culturas. Esses métodos utilizam fornecimento de calor ao caule atuando como "marcadores" da seiva, o qual pode ser relacionado com a densidade de fluxo de seiva e, por consequência, com a transpiração (Rojas et al., 2007).

O método de dissipação de calor foi desenvolvido, originalmente, para medida de fluxo de seiva em espécies florestais, por Granier \& Berger (1986). A sonda de dissipação térmica utiliza princípios físicos relativamente simples que podem ser aplicados diretamente no campo, sem alterar as condições fisiológicas e microclimáticas da planta. Granier (1985) relacionou a velocidade de dissipação de calor aplicado em um ponto do tronco com o fluxo de seiva. A variação temporal da diferença de temperatura entre o ponto acima e o ponto abaixo (10 $\mathrm{cm}$ aproximadamente) é ocasionada pelo transporte convectivo de calor pela seiva (Rojas, 2003).

Portanto, no período de um dia a máxima diferença de temperatura entre os dois pontos de medida significa que o fluxo de seiva é mínimo ou nulo enquanto a mínima diferença significa uma taxa máxima de fluxo de seiva através dessa área de transporte. A máxima diferença de temperatura acontece normalmente no amanhecer enquanto a mínima, ao entardecer.

Granier (1985) calibrou o método em laboratório, primeiro com três espécies de plantas lenhosas e, posteriormente, com seis, verificando que uma única equação de calibração pode ser utilizada para todas elas. Em função disto, Smith \& Allen (1996), também propuseram uma equação que pode ser usada em qualquer planta lenhosa com diâmetro de caule (diâmetro na altura do peito) superior a $4 \mathrm{~cm}$. Vários autores já testaram esse método em condições naturais de campo, em numerosas espécies de plantas lenhosas demonstrando ser este método uma alternativa promissora na medida da transpiração nessas plantas (Granier, 1987; Ferreira \& Zitscher, 1996; Granier \& Bréda, 1996; Loustau et al., 1998; Tournebize \& Boistard, 1998; Naves-Barbiero et al., 2000; Hubbard et al., 2004; Tatarinov et al., 2005),

Comparado com os outros dois métodos térmicos mais comumente usados, o de pulso de calor (Green \& Clothier, 1988; Köstner et al., 1998; Giorio \& Giorio, 2003) e o de balanço de calor no caule (Steinberg et al., 1989; Baker \& Nieber, 1989; Angelocci \& Valancogne, 1993), o método da sonda de dissipação térmica é o mais simples quanto à instrumentação utilizada, além de apresentar facilidade de confecção e instalação da sonda, bem como versatilidade quanto à adaptação para qualquer planta lenhosa com diâmetro de caule maior que $4 \mathrm{~cm}$ (Smith \& Allen, 1996).

Ferreira \& Zitscher (1996) observaram que uma das principais fontes de erro deste método da dissipação térmica pode ser atribuída ao gradiente natural da temperatura no tronco, principalmente quando o sensor é colocado muito próximo ao solo. O referido gradiente térmico natural é o efeito da radiação e da temperatura do ar e do solo que atuam no caule, afetando o valor da medida térmica do sensor que, por sua vez, acaba afetando significativamente a estimativa do fluxo de seiva. Com efeito, Granier (1987) já tinha observado diferença entre os valores de fluxo de seiva medidos com três sensores diferentes, orientados em lados opostos, $\operatorname{com} 120^{\circ} \mathrm{em}$ volta do tronco e na altura de $1,0 \mathrm{~m}$ acima do solo. Loustau et al. (1998) também observaram discrepâncias entre os valores de fluxo de seiva medidos em duas alturas do caule (1,5 e 8,5 m) em uma planta de pinus devido, possivelmente, à existência desse gradiente natural.

O método da sonda de dissipação de calor só possibilita a determinação da densidade de fluxo de seiva na secção transversal do caule e sua transformação em fluxo de água exige a determinação mais exata possível da área condutiva de seiva no lenho (alburno).

A determinação da área efetiva do xilema é uma fonte de erro no método e, ao exigir a retirada de uma amostra do lenho ou a destruição total da planta, torna-se um procedimento desvantajoso desse método em relação ao de balanço de calor. Por outro lado, a grande vantagem do método é a facilidade de confecção e instalação do sensor quando comparado com outros métodos térmicos (Braun, 1997). Outra fonte de erro é representada pelas variações espaciais da densidade de fluxo de seiva no lenho, que exige a adequada instalação da sonda (Lu et al., 2000).

As medidas de transpiração constituem elemento essencial para compreensão da fisiologia da árvore e a dinâmica da transferência de água em experimentos florestais (Granier, 1985). Estimativas de evaporação para as regiões com cobertura vegetal heterogênea são de grande importância para o problema do dimensionamento de energia, água, dinâmica e fluxos (Veen et al., 1991) que têm sido alvo de muitos estudos.

O objetivo do estudo foi analisar o comportamento do fluxo de seiva em espécies da floresta de transição Amazônia Cerrado e caracterizar a dependência do fluxo de seiva em função do déficit de pressão de vapor da atmosfera (DPV) em diferentes estações do ano na floresta de transição Amazônica Cerrado.

\section{Material e Métodos}

O estudo foi realizado a $50 \mathrm{~km}$ a nordeste de Sinop $\left(11^{\circ}\right.$ 24' 43,4" S: 55' '19' 25,7" O), Mato Grosso, Brasil, a 423 m acima do nível do mar, onde se encontra instalada uma torre micrometeorológica de $42 \mathrm{~m}$ de altura pertencente ao Programa de Pós-Graduação em Física Ambiental da Universidade Federal do Mato Grosso (UFMT), que participa do projeto Experimento de Grande Escala da Biosfera-Atmosfera na Amazônia (LBA) (Figura 1).

A área de estudo é constituída por uma floresta tropical de transição, que ocupa o ecótono entre os biomas Floresta Amazônica e Cerrado (Ackerly et al., 1989). Conforme classificação de Köppen (Vianello \& Alves, 1991) o clima nessa região é do tipo $\mathrm{AW}$, com pequena variação sazonal na temperatura, com maior variação no padrão de precipitação, sendo caracterizado por dois períodos bem definidos: seco (junho a setembro) e úmido (dezembro a março), apresentando dois períodos de transição, um seco/úmido (outubro e novembro) e um úmido/seco (abril e maio) (Vourlitis et al., 2005). 


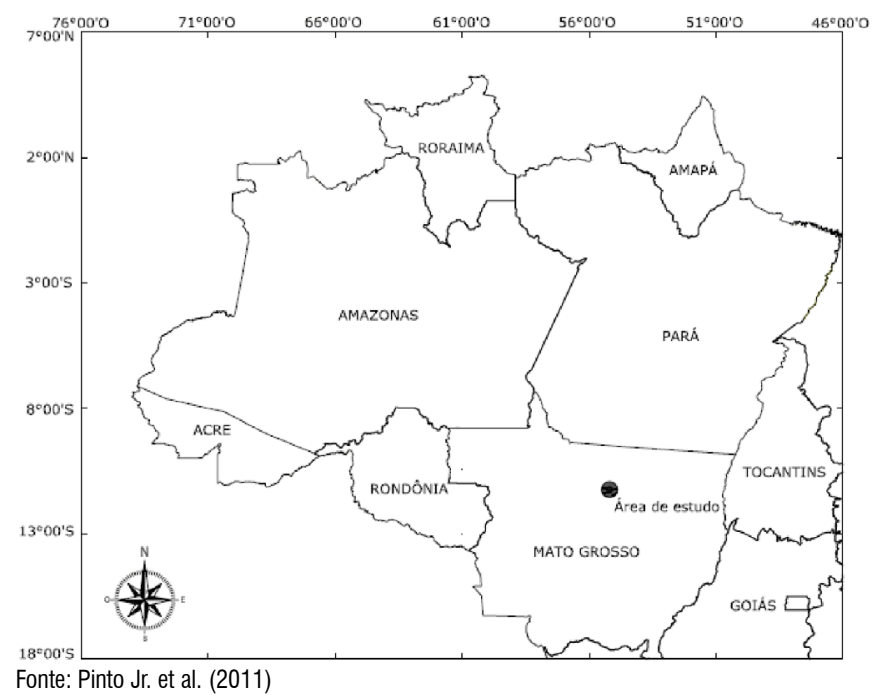

Figura 1. Mapa de localização da área de estudo

O solo da área experimental foi classificado como Neossolo quartzarênico-órtico do tipo A moderado e álico, com profundidade de $50 \mathrm{~cm}$ e textura arenosa, com $83,6 \%$ de areia, $4,4 \%$ de silte e $2,2 \%$ de argila (Priante Filho et al., 2004). Esses solos, além de bastante arenosos, são pobres em nutrientes, têm alta porosidade e drenam rapidamente a saturação provocada por fortes chuvas (Vourlitis et al., 2002).

A vegetação é classificada como floresta de transição ombrófila estacional (Vilani et al., 2006) e constituída por espécies arbóreas cuja altura média varia de 28-30 m. Essas espécies são típicas de floresta amazônica (Ackerly et al., 1989, Lorenzi, 2000; 2002), tendo, como principais representantes, as espécies Tovomita schomburgkii Planch e Triana, Brosimum lactescens (S. Moore) C.C. Berg, Qualea paraensis Ducke (Rainfor, J. Lloyd, Universityof Leeds), Vochysia sp., Ocote aspixiana, Dinizia excelsa, Quina pteridophilla e Mezilaurus sitauba (Vourlitis et al., 2002; 2005). A área possui cerca de 80 espécies distribuídas em 35 famílias sendo que, em torno de $50 \%$, a floresta está representada pelas famílias Burseraceae (P. sagotianum), Clusiaceae ( $T$. schomburgkii), e Moraceae ( $B$. lactescens) (Vourlitis et al., 2008).

As espécies utilizadas no estudo estão ilustradas na Tabela 1 procurando-se verificar o fluxo de seiva em diferentes espécies, de variados diâmetros e alturas.

Tabela 1. Espécies utilizadas na medida do fluxo de seiva, na floresta de transição Amazônia-Cerrado

\begin{tabular}{lccc}
\hline \multicolumn{1}{c}{ Espécie } & $\begin{array}{c}\text { Altura } \\
\text { (m) }\end{array}$ & $\begin{array}{c}\text { DAP } \\
\text { (cm) }\end{array}$ & $\begin{array}{c}\mathbf{N}^{\mathbf{0}} \text { de } \\
\text { indivíduos }\end{array}$ \\
Brosimumlactescens (S. Moore) C. C. Berg & 28 & 58 & 1 \\
Qualeaparaensis Ducke & 25 & 44 & 1 \\
Tovomitaschomburgkii Planch e Triana & 20 & 16 & 4 \\
Cordiaalliodora (Ruiz e Pav.) Oken & 15 & 40 & 1 \\
Protiumsagotianum Marchland & 10 & 12 & 2 \\
\hline
\end{tabular}

Fonte: Vourlitis et al. (2008); DAP - diâmetro da altura do peito

Seguindo a classificação proposta por Morellato et al. (1989) quanto aos padrões de produção e queda das folhas, a espécie em estudo está incluída na categoria semi-decídua e possui o seguinte comportamento: a) em meados de março surgem as gemas apicais; b) ocorre a expansão de duas folhas quando começam as chuvas, em setembro; c) logo que as chuvas se tornam mais frequentes e intensas, há uma explosão do crescimento do ramo e um aumento do número de folhas expandidas; d) em novembro pode-se observar que as folhas jovens estão totalmente expandidas e algumas folhas velhas (que surgiram no ano anterior) estão em processo de senescência, tendo-se observado um processo de abscisão foliar mais intenso no período da seca até o início de agosto.

O sistema de medida do fluxo de seiva envolve a inserção perpendicular no tronco de duas sondas distanciadas $4 \mathrm{~cm}$ entre si 4, na linha vertical do tronco. A sonda superior é aquecida de forma constante com fornecimento de potência elétrica de $0,2 \mathrm{~W}$. A variação da diferença de temperatura entre a sonda aquecida (superior) e a não aquecida (inferior) em cada instante é determinada pelo transporte convectivo de calor através da seiva. Assim, a máxima diferença entre as duas sondas significa que o fluxo de seiva é mínimo ou nulo enquanto a mínima diferença significa uma taxa máxima de fluxo de seiva dessa área de transporte (Granier, 1987).

A sonda instalada do presente experimento foi o modelo TDP $30 \mathrm{~mm}$ (Dynamax, Huston, TX), confeccionada com fio de constantan de diâmetro de $0,5 \mathrm{~mm}$. A inserção foi feita utilizando-se brocas de diâmetro superior ao diâmetro do fio; logo após foi introduzida uma massa (tipo calafetar), a fim de que não houvesse interferência externa da temperatura; posteriormente, a árvore foi encoberta por uma cápsula térmica para evitar o efeito da incidência da radiação sobre as medidas do fluxo de seiva. Em todos os espécimes todos os sensores foram instalados na altura no peito.

Para as espécies em estudo (Tabela 1), usou-se a Eq. 1, para estabelecer o índice de fluxo de seiva $\left(\mathrm{k}_{\mathrm{s}}\right)$ :

$$
\mathrm{k}_{\mathrm{s}}=\frac{\left(\Delta \mathrm{T}_{\max }-\Delta \mathrm{T}_{\text {inst }}\right)}{\Delta \mathrm{T}_{\text {inst }}}
$$

em que:

$\Delta \mathrm{T}_{\max }$ - diferença máxima de temperatura entre dois pontos de medida

$\Delta \mathrm{T}_{\text {inst }}$ - diferença de temperatura medida instantaneamente

Posteriormente foi calculada a velocidade do fluxo de seiva ( $\mathrm{cm} \mathrm{s}^{-1}$ ), usando-se a Eq. 2.

$$
\mathrm{V}=0,0019 \mathrm{k}_{\mathrm{s}}^{1,231}
$$

em seguida, o fluxo de seiva (FS) $\left(\mathrm{kg} \mathrm{h}^{-1}\right)$ é estimado com base na Eq. 3.

$$
\mathrm{FS}=\mathrm{V}(\mathrm{SA})
$$

em que:

AS - área do alburno na altura do peito, normalmente considerada a área ocupada pelo xilema

A área do xilema ocupado foi calculada levando-se em consideração os seguintes procedimentos:

O diâmetro na altura do peito $(\mathrm{cm})$ de cada espécie, conforme a Eq. 4. 


$$
\mathrm{D}=\frac{\mathrm{P}}{\pi} \times 100
$$

em que:

$\mathrm{P} \quad$ - perímetro da circunferência da árvore na altura do peito, dado em centímetros e $\pi=3,14159$

A partir deste calculou-se a área total do tronco na altura do peito $\left(\mathrm{m}^{2}\right)$, de acordo com a Eq. 5 .

$$
\mathrm{A}=\frac{\pi \times \mathrm{D}^{2}}{4}
$$

O diâmetro na altura do peito foi calculado para que desconsiderasse a casca da árvore, conforme a Eq. 6:

$$
\mathrm{D}_{1}=\mathrm{D}-(\mathrm{C} \times 2)
$$

em que:

D - diâmetro da árvore e C é a casca da árvore, $\mathrm{cm}$

A área na altura do peito foi novamente calculada considerando-se o diâmetro acima.

$$
\mathrm{A}_{1}=\frac{\pi \times \mathrm{D}_{1}^{2}}{4}
$$

O diâmetro na altura do peito foi novamente recalculado considerando-se a área ocupada pelo xilema, sendo a amostra obtida com auxílio de um Increment borer, que é uma ferramenta especializada utilizada para extrair uma secção de madeira ou tecido de uma árvore com mínimo prejuízo.

Foi retirada uma amostra de cada espécie, a fim de que se pudesse estimar o tamanho do alburno de cada amostra.

$\mathrm{O}$ diâmetro na altura do peito foi calculado usando-se a seguinte Eq. 8.

$$
\mathrm{D}_{2}=\mathrm{D}-(\mathrm{X} \times 2)
$$

em que:

D - diâmetro na altura do peito da árvore

X - distância radial do xilema do tronco da árvore, $\mathrm{cm}$

Aárea na altura do peito foi novamente calculada levando-se em consideração o diâmetro acima.

$$
\mathrm{A}_{2}=\frac{\pi \times \mathrm{D}_{2}^{2}}{4}
$$

A partir de então, a área do alburno na altura do peito (SA) foi calculada usando-se a diferença entre as duas áreas, conforme a Eq. 10:

$$
\mathrm{SA}=\mathrm{A}_{1}-\mathrm{A}_{2}
$$

O déficit de pressão de vapor foi calculado pela diferença entre a pressão de vapor d'água do ar saturado $\left(\mathrm{e}_{\mathrm{s}}\right)$ e a pressão de vapor d'água do ar atual (e) usando-se a temperatura e a umidade da torre meteorológica da altura de $20 \mathrm{~m}$ entre os períodos de Outubro de 2007 a Outubro de 2008.

A pressão de vapor d'água do ar saturado $\left(\mathrm{e}_{\mathrm{s}}\right)(\mathrm{kPa})$ para cada altura foi calculada como função da temperatura do $\operatorname{ar}\left(\mathrm{T}_{\mathrm{ar}}\right)$ em cada altura, conforme a Eq. 11 .

$$
\mathrm{e}_{\mathrm{s}}=0,6108 \times 10^{\left(\frac{7,5 \mathrm{~T}_{\mathrm{ar}}}{237,3+\mathrm{T}_{\mathrm{ar}}}\right)}
$$

A pressão de vapor d'água do ar atual (e) foi calculada pelo produto da pressão de vapor d'água do ar saturado $\left(\mathrm{e}_{\mathrm{s}}\right) \mathrm{e}$ a umidade relativa do ar, definida na Eq. 12.

$$
\mathrm{e}=\mathrm{e}_{\mathrm{s}} \mathrm{UR}
$$

\section{Resultados e Discussão}

Durante o período experimental as curvas de variação sazonal do fluxo de seiva evidenciam a ocorrência de picos no período úmido. Os valores máximos diários de fluxo de seiva foram observados na primeira metade do período úmido, quando o potencial evaporativo da atmosfera era baixo (conjugação de valores baixos de radiação global e de déficit de pressão de vapor de água) quando não há restrições hídricas no solo (devido à ocorrência de precipitação). Na floresta de transição Amazônia - Cerrado, o valor máximo do fluxo de seiva foi de $227 \mathrm{~L} \mathrm{~d}^{-1}$ no período de transição seco-úmido; a partir daí, o fluxo de seiva decresce progressivamente atingindo seu valor mínimo no período úmido, de $0,18 \mathrm{~L} \mathrm{~d}^{-1}$. No início do período de transição seco-úmido observou-se, após as primeiras chuvas, recuperação dos fluxos de seiva. Esta mesma tendência de variação sazonal do fluxo de seiva foi evidenciada pela relação entre valores diários de transpiração e do déficit de pressão de vapor (DPV).

Do ponto de vista da contribuição relativa das espécies em que se mediu o fluxo de seiva sobre a evapotranspiração da floresta considerando-se este fluxo de seiva como representativo da taxa transpiratória pode-se inferir que, pelo menos para as espécies de estudo, não existe evidência de que haja, durante o período seco do ano, algum incremento na taxa transpiratória por unidade de área foliar. Esperava-se que as plantas obtivessem maior incremento transpiratório, o que não ocorreu porque todas, com exceção da $B$. lactescens, tiveram redução no fluxo de seiva no período seco, conforme se observa na Tabela 2. A umidade do solo na área de estudo teve comportamento sazonal, ou seja, acompanhando o regime pluviométrico, sendo que a maior concentração de água no solo se fez presente no mês de fevereiro, com $0,156 \mathrm{~m}^{3} \mathrm{~m}^{-3}$ e a menor em agosto, com $0,075 \mathrm{~m}^{3} \mathrm{~m}^{-3}$ (Figura 2).

Bucci et al. (2008) apresentaram, em diferentes fisionomias de Cerrado em Brasília, transpiração vegetal, medida por meio da técnica do fluxo de seiva, valores similares entre os períodos seco e úmido, com valores de $0,20 \mathrm{~mm} \mathrm{~d}^{-1}\left(17,20 \mathrm{~L} \mathrm{~d}^{-1}\right)$ nas áreas de cerrado aberto, além de valores próximos a $0,80 \mathrm{~mm}$ $\mathrm{d}^{-1}\left(69,12 \mathrm{~L} \mathrm{~d}^{-1}\right)$ no cerrado denso e no cerradão. Em estudos realizados por Costa \& Marenco (2007), na floresta tropical Amazônica, foram registrados valores médios superiores aos 
Tabela 2. Médias e intervalos de confiança (superior e inferior)

\begin{tabular}{|c|c|c|c|c|}
\hline \multirow{3}{*}{ Espécie } & \multicolumn{4}{|c|}{ Médias por período do fluxo de seiva $\left(\mathrm{L} \mathrm{d}^{-1}\right)$} \\
\hline & \multirow{2}{*}{ Período } & \multirow{2}{*}{ Média } & \multicolumn{2}{|c|}{ Limite } \\
\hline & & & Superior & Inferior \\
\hline \multirow{4}{*}{ B. lactescens } & Seco & $50,98 \mathrm{Aa}$ & 66,35 & 35,59 \\
\hline & Seco-úmido & 52,19 Aab & 69,97 & 35,84 \\
\hline & Úmido & $61,67 \mathrm{Ab}$ & 74,57 & 49,84 \\
\hline & Úmido-seco & $47,95 \mathrm{Aa}$ & 63,23 & 32,86 \\
\hline \multirow{4}{*}{ Q. paraensis } & Seco & $51,83 \mathrm{ABa}$ & 66,09 & 37,34 \\
\hline & Seco-úmido & 53,90 ABab & 70,53 & 37,70 \\
\hline & Úmido & $61,65 \mathrm{Ab}$ & 70,00 & 53,21 \\
\hline & Úmido-seco & $35,03 \mathrm{Bab}$ & 46,19 & 25,41 \\
\hline \multirow{4}{*}{ T. schomburgkii } & Seco & $47,60 \mathrm{Ca}$ & 62,17 & 35,09 \\
\hline & Seco-úmido & $74,75 \mathrm{Ba}$ & 86,94 & 63,84 \\
\hline & Úmido & $183,74 \mathrm{Aa}$ & 194,83 & 172,46 \\
\hline & Úmido-seco & $21,82 \mathrm{Db}$ & 28,22 & 15,85 \\
\hline \multirow{4}{*}{ C. alliodora } & Seco & $7,47 \mathrm{Cb}$ & 9,78 & 5,24 \\
\hline & Seco-úmido & $52,75 \mathrm{Bb}$ & 56,92 & 48,97 \\
\hline & Úmido & $172,65 \mathrm{Aa}$ & 181,25 & 164,68 \\
\hline & Úmido-seco & $8,77 \mathrm{Cc}$ & 10,34 & 7,33 \\
\hline \multirow{4}{*}{ P. sagotianum } & Seco & 3,54 Dc & 4,76 & 2,35 \\
\hline & Seco-úmido & $29,33 \mathrm{Ac}$ & 32,45 & 26,65 \\
\hline & Úmido & $15,35 \mathrm{Bc}$ & 15,96 & 14,76 \\
\hline & Úmido-seco & 6,72 Cc & 7,69 & 5,75 \\
\hline
\end{tabular}

Para uma mesma espécie as médias seguidas de mesma letra maiúscula nas colunas não diferem entre si ao passo que, para o mesmo período do ano, as médias seguidas de mesma letra minúscula não diferem entre si, em ambos os casos, considerando-se os limites superior e inferior da média obtidos por bootstrap para fins de comparação, a nível de 0,05 de probabilidade

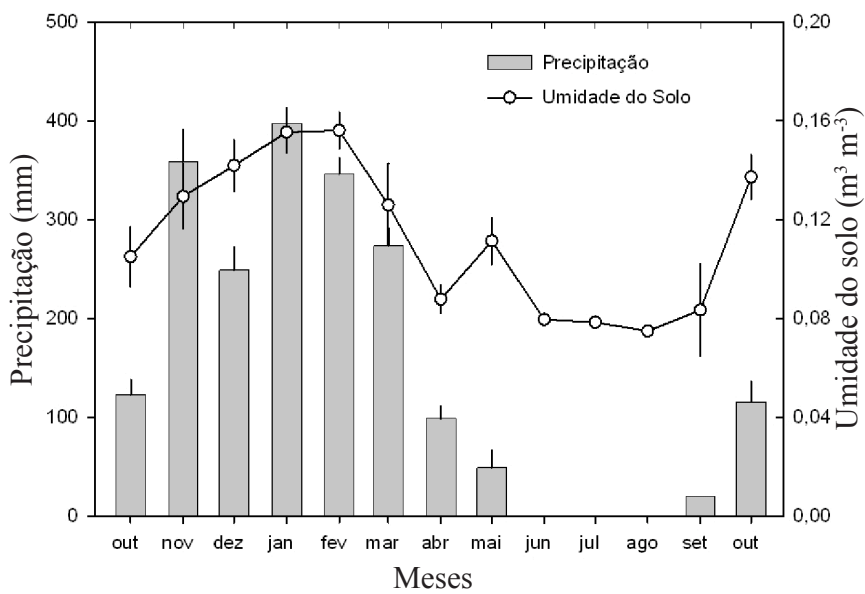

Figura 2. Precipitação acumulada no mês $( \pm D P)$ e umidade (média \pm DP) do solo na floresta de transição Amazônica Cerrado, de outubro de 2007 a outubro de 2008

do presente estudo, sendo que para o período úmido os valores de fluxo de seiva foram de $170 \mathrm{~L} \mathrm{~d}^{-1}$, o que se deve às variações das espécies em estudo (porte, diâmetro, IAF, entre outros) e das condições climáticas que diferem do presente estudo.

No início do período luminoso nota-se uma relação positiva entre o aumento de DPV e o fluxo de seiva, até o fluxo de seiva atingir um valor máximo, existindo um valor limite

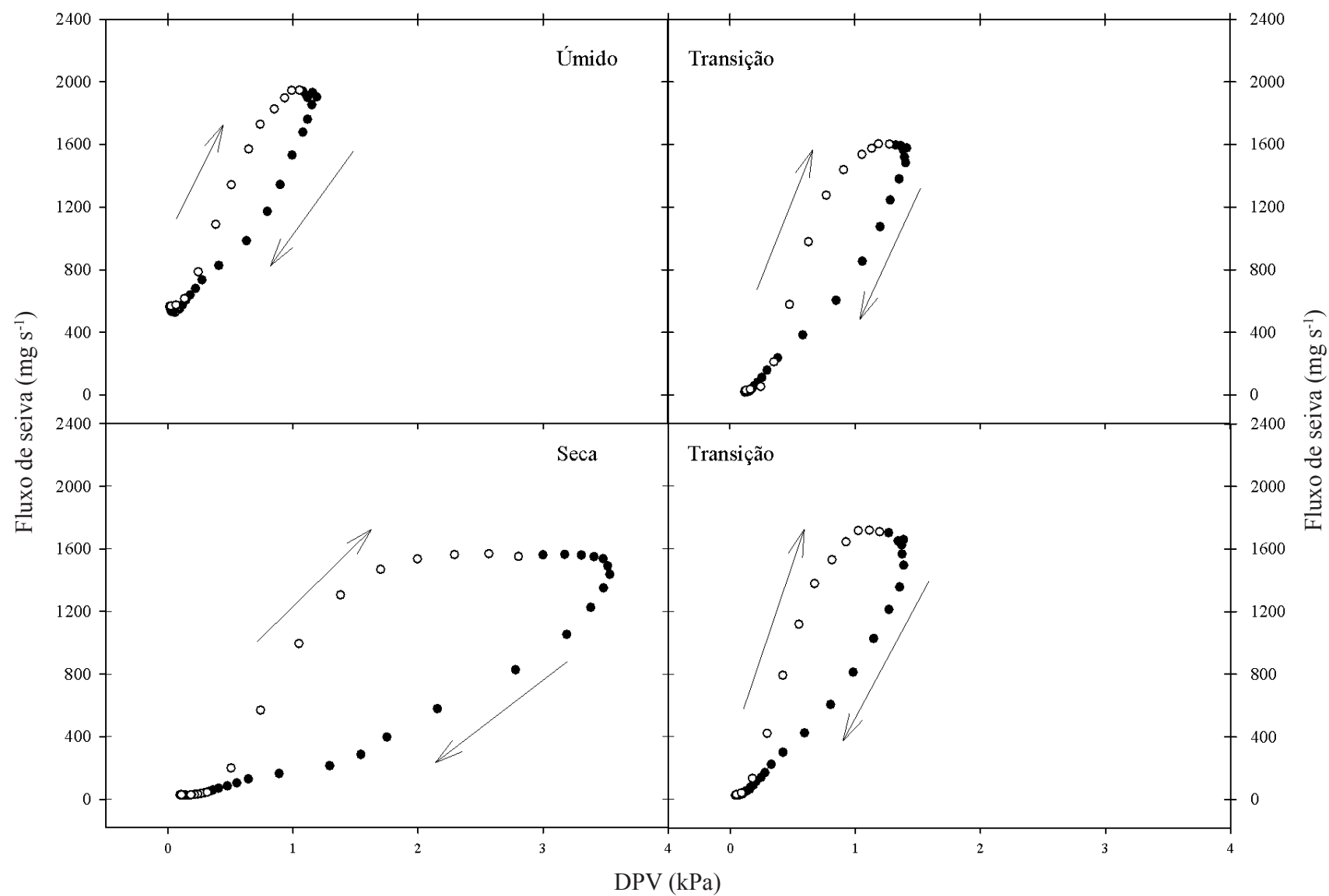

Os símbolos em aberto correspondem aos valores obtidos antes das $12 \mathrm{~h}$ enquanto os símbolos fechados, a valores obtidos após as $12 \mathrm{~h}$. A direção das setas corresponde ao curso de aumento ou decréscimo do DPV, ao longo do dia

Figura 3. Relação entre o fluxo de seiva e o déficit de pressão de vapor para Brosimum lactescens (S. Moore), nos períodos úmido, de transição úmido/seco, seco e de transição seco/úmido 
de DPV, correspondente ao fluxo de seiva máximo. A partir desse valor limite de DPV, o fluxo de seiva diminui, apesar da demanda evaporativa continuar aumentando, refletindo o controle estomático no processo da transpiração. Devido ao controle estomático esta redução de fluxo de seiva varia de estação para estação, sendo que nas estações úmidas e as transições úmida-seca e seca-úmida ocorrem por volta do meio-dia e se mantêm ao logo do período da tarde. $\mathrm{O}$ valor limite de DPV é, nessas estações, de 1 a $1,5 \mathrm{kPa}$; na estação seca o comportamento do fluxo de seiva ocorre por volta das $14 \mathrm{~h}$, devido ao controle estomático e o valor limite de DPV é maior quando comparado com o das outras estações, ou seja, por volta de $2,8 \mathrm{kPa}$ (Figura 3 ).

\section{Conclusões}

1. A redução do fluxo de seiva no período seco do ano não confirma a hipótese da manutenção do processo de evapotranspiração com significativa contribuição da transpiração das plantas.

2. O fluxo de seiva nas espécies estudadas depende mais das condições atmosféricas do que da disponibilidade de água no solo, o que suscita um possível armazenamento da água no caule, nos períodos mais secos.

\section{Agradecimentos}

Agradecemos aos órgãos de fomento de Pesquisa: CAPES, CNPq e FAPEMAT e ao Experimento de Grande Escala da Biosfera-Atmosfera na Amazônia - LBA

\section{Literatura Citada}

Ackerly, D. D.;Thomas, W. W.; Ferreira, C. A. C.; Pirani, J. R. The forest-cerrado transition zone in southern Amazonia: Results of the 1985 Projecto Flora Amazonica expedition to Mato Grosso. Brittonia, v.41, p.113-128,1989.

Angelocci, L. R.; Valancogne, C. Leaf area and water flux in apple trees. Journal of Horticultural Science, v.68, p.299307, 1993.

Baker, J. M.; Nieber, J. L. An analysis of the steady-state heat balance method for measuring sap flow in plants. Agricultural and Forestry Meteorology, v.48, p.93-109, 1989.

Braun, P. Sap flow measurements in fruit trees - Advantages and shortfalls of currently used systems. Acta Horticultural, v.449, p.267-272, 1997.

Bucci, S. J.; Sholz, F. G.; Goldstein, G.; Hoffmann, W. A.; Meinzer, F. C.; Franco, A. C.; Giambelluca, T.; Wilhelm, F. M. Controls on stand transpiration and soil water utilization along a tree density gradient in a Neotropical savanna. Agriculturaland Forest Meteorology, v.148, p.839-849, 2008.

Costa, G. F.; Marenco, R. A. Fotossíntese, condutância estomática e potencial hídrico foliar em árvores jovens de andiroba (Carapaguianensis). Acta Amazonica, v.37, p.229-234, 2007.
Dynamax. A thermal dissipation sap velocity probe for measurement of sap flow in plants, Huston: Dynamax, Inc., 1997. 34p.

Ferreira, M. I.; Zitscher, H. Measurements performed with Granier method in short trunks near the soil. In: Workshop on Measuring Sap Flow in Intact Plants, 3, 1996, Barcelona. Proceedings... Barcelona: Sitges, 1996. p.1-3.

Giorio, P.; Giorio, G. Sap flow of several olive trees estimated with the heat-pulse technique by continuous monitoring of a single gauge. Environmental and Experimental Botany, v.49, p.9-20, 2003.

Granier, A. Une nouvelle method pour la mesure du flux de sève brute dans le tronc des arbres. Annales des Sciences Forestières, v.42, p.193-200, 1985.

Granier, A. Mesure du flux de sève brute dans le tronc du douglas par une nouvelle method thermique. Annales des Sciences Forestierès, v.44, p.1-14, 1987.

Granier, A.; Bréda, N. Modeling canopy conductance and stand transpiration of an oak forest from sap flow measurements. Annales des Sciences Forestières, v.53, p.537-546, 1996.

Granier, E.; Berger, A. Effect of water stress on stem diameter changes of peach trees growing in the field.Journal of Applied Ecology, v.23, p.193-209, 1986.

Green, S. R.; Clothier, B. E. Water use of kiwifruit vines and apples trees by the heat-pulse technique.Journal of Experimental Botany, v.39, p.115-123, 1988.

Hubbard, R. M.; Ryan, M. G.; Giardina, C. P.; Barnard, H.The effect of fertilization on sap flux and canopy conductance in a Eucalyptus saligna experimental forest. Global Change Biology, v.10, p.427-136, 2004.

Köstner, B.; Granier, A.; Cermák, J. Sapflow measurements in forest stands: methods and uncertainties. Annuary Science Forest, v.55, p.13-27, 1998.

Lorenzi, H. Árvores brasileiras, v.1. São Paulo: Instituto Plantarum de Estudos da Flora, Ltd., 2000. 384p.

Lorenzi, H. Árvores brasileiras, v.2. São Paulo: Instituto Plantarum de Estudos da Flora, Ltd., 2002. 384p.

Loustau, D.; Domec, J. C.; Bosc, A. Interpreting the variations in xylem sap flux density within the trunk of maritime pine (PinuspinasterAit.): Application of a model for calculating water flows at tree and stand levels. Annales des Sciences Forestierès, v.55, p.29-40, 1998.

Lu, P.; Müller, W. J.; Chacro, E. K. Spatial variations in xylem sap flux density in the trunk of orchard-grown, mature mango trees under changing soil water conditions. Tree Physiology, v.20, p.683-692, 2000.

Morellato, L. P. C.; Rodrigues, R. R.; LeitãoFilho, H. F.; Joly, C. A. Estudo comparativo de fenologia d espécies arbóreas de florestas de altitude e mesófila semidecídua na Serra do Japi, Jundiaí, São Paulo. Revista Brasileira de Botânica, v.12, p.85-89, 1989.

Naves-Barbiero, C. C.; Franco, A. C.; Bucci, S. J.; Goldstein, G. Fluxo de seiva e condutância estomática de duas espécies lenhosas sempre-verdes no campo sujo e cerradão. Revista Brasileira de Fisiologia Vegetal, v.12, p.119-134, 2000.

Pinto Jr., O. B.; Sanches, L.; Lobo, F. A.; Brandão, A. A.; Nogueira, J. S. Leaf area index of a tropical semi-deciduous forest of the Southern Amazon Basin. International Journal of Biometeorology, v.55, p.109-118, 2011. 
Priante Filho, N.; Hayashi, M. M. S.; Nogueira, J. S. de; Campelo Jr., J. H.; Nunes, P. C.; Sanches, L.; Couto, E. G.; Hoeger, W.; Raiter, F.; Trienweiler, J. L.; Miranda, E. J.; Priante, P. C.; Pereira, L. C.; Biudes, M. S.; Fritzen, C. L.; Lacerda, M.; Suli, G. S.; Shiraiwa, S.; Silveira, M.; Vourlitis, G. L. Comparison of the mass and energy exchange of a pasture and a mature transitional tropical forest of the southern Amazon basin during the wet-dry season transition. Global Change Biology, v.10, p.863-876, 2004.

Rojas, J. S. D. Avaliação do uso do fluxo de seiva e da variação do diâmetro do caule e de ramos na determinação das condições hídricas de citros, como base para o manejo de irrigação. Piracicaba: ESALQ/USP, 110p. 2003. Tese Doutorado

Rojas, J. S. D.; Angelocci, L. R.; Folegatti, M. V.; Coelho Filho, M. A. Desempenho da sonda de dissipação térmica na medida da transpiração de plantas jovens de lima ácida. Engenharia Agrícola, v.27, p.404-413. 2007.

Smith, D. M.; Allen, S. J. Measurement of sap flow in plants stems. Journal of Experimental Botany, v.47, p.1833-1844, 1996.

Steinberg, S. L.; Bavel, C. H. M. van; McFarland, M.A gauge to measure mass flow rate of sap in stems and trunks of woody plants. Journal of the American Society for Horticultural Science, v.114, p.466-472, 1989.

Tatarinov, F.; Kucera, J.; Cienciala, E. The analysis of physical background of tree sap flow measurement based on thermal methods. Measurement Science Technology, v.16, p.11571169, 2005.

Tournebize, R.; Boistard, S. Comparison of two sap flow methods for the estimation of tree transpiration.Annales des Sciences Forestieres, v.55, p.706-713, 1998.
Veen, A. W. L.; Hutjes, R. W. A.; Klaassen, W.; Kruijt, B.; Lankreijer, H. J. M. Evaporative conditions across a grassforest boundary: A comment on the strategy for regionalizing evaporation. In Kienitz, G.;Milly, P. C. D.; Genuchten, M. Th. van; Rosbjerg, D.; Shuttleworth, W. J. (ed.), Hydrological interactions between atmosphere, soil and vegetation. International Association of Hydrological Sciences Publication n.204. Wallingford: IAHS Press, 1991. p.43-52.

Vianello, R. L.; Alves, A. R. Meteorologia básica e aplicações. Viçosa: UFV, 1991. 449p.

Vilani, M. T.; Sanches, L.; Nogueira, J. S.; Priante-Filho, N. Sazonalidade da radiação, temperatura e umidade em uma floresta de transição Amazônia Cerrado. Revista Brasileira de Meteorologia, v.21, p.119-131, 2006.

Vourlitis, G. L., Nogueira, J. S.; Lobo, F. A.; Sendall, K. M.; Paulo S. R.; Dias, C. A. A.; Pinto Júnior, O. B.; Andrade, N. L. R. Energy balance and canopy conductance of a tropical semi-deciduous forest of the southern Amazon Basin, Water Resources Research, v.44, W03412, doi: 10.1029/2006wr005526, 2008.

Vourlitis, G. L.; Nogueira, J. de S.; Priante Filho, N.; Hoeger, W.; Raiter, F.; Biudes, M. S.; Arruda, J. C.; Capistrano, V. B.; Faria, J. L. B. de; Lobo, F. de. A.The sensitivity of diel $\mathrm{CO}_{2}$ and $\mathrm{H}_{2} \mathrm{O}$ vapor exchange of a tropical transitional forest to seasonal variation in meteorology and water availability, Earth Interactions, v.9, p.1-23, 2005.

Vourlitis, G. L.; PrianteFilho, N.; Hayashi, M. M. S.; Nogueira, J. S; Caseiro, F. T. Campelo Jr., J. H. Seasonal variations in the evapotranspiration of a transitional tropical forest of Mato Grosso, Brazil. Water Resources Research, v.38, p.30.1-30.11, 2002. 